\title{
Sistema metodológico integral del aprendizaje del Derecho Constitucional a partir de la vivencia: una red de estrategias didácticas
}

\section{Comprehensive methodological system of Constitutional Law learning from the experience: a network of teaching strategies.}

\author{
Katherine Becerra Valdivia ${ }^{1}$ \\ Carolina Salas Salazar ${ }^{2}$
}

\begin{abstract}
Resumen: La evolución del Derecho Constitucional en los últimos años ha transformado la manera de comprender el rol que cumplen las constituciones en los sistemas jurídicos, y Chile no ha sido la excepción. Como primera aproximación, podemos señalar que la comprensión actual de estos documentos como norma jurídica vinculante ha originado una profusa jurisprudencia sobre una gran cantidad de temas que afectan a toda la sociedad. Sin lugar a dudas, que esta visión impacta necesariamente en la enseñanza y formación de los futuros abogados, es por ello que el objetivo que persigue esta ponencia es exponer nuestra experiencia en este sentido, específicamente en la utilización de "la vivencia" como estrategia de enseñanza - aprendizaje para la disciplina del Derecho Constitucional, sus ventajas y desventajas.
\end{abstract}

Palabras Clave: vivencia - enseñanza - aprendizaje

Abstract: The evolution of constitutional law in recent years has transformed the way of understanding the role that constitutions in legal systems, and Chile has been no exception. As a first point of view, it is argued that the current understanding of these documents -as binding legal norm- has triggered a prolific jurisprudence on a great number of issues that affect all of society. Undoubtedly, this view impacts necessarily on teaching and training of future lawyers. Thereby, this paper aims to report our practice in this sense; specifically, on the use of "the teaching experience" as a strategy of teaching - learning to the discipline of Constitutional Law, considering its advantages and disadvantages.

Keywords: experience - teaching of Constitutional law - learning from the experience.

\footnotetext{
${ }^{1}$ Abogada. Magíster en Derecho por la Universidad Católica del Norte. Profesora de Derecho Constitucional de la Facultad de Ciencias Jurídicas de la Universidad Católica del Norte, sede Coquimbo. Correo electrónico: kbecerra@ucn.cl

${ }^{2}$ Abogada. Doctora en Derecho por la Universidad de Castilla-La Mancha (España). Profesora de Derecho Constitucional de la Facultad de Ciencias Jurídicas de la Universidad Católica del Norte, sede Coquimbo. Correo electrónico: csalas@ucn.cl
} 


\section{Introducción}

El artículo 9 de la Conferencia Mundial sobre la Educación Superior señala que "en un mundo en rápido cambio, se percibe la necesidad de una nueva visión y un nuevo modelo de enseñanza superior, que debería estar centrado en el estudiante" 3 , lo que conlleva la necesidad de replantear la relación docente - estudiante.

Asimismo, en el informe emitido a la UNESCO por la Comisión Internacional sobre la Educación para el Siglo XXI, se reconocía que la educación "tiene la misión de permitir a todos sin excepción hacer fructificar todos sus talentos y todas sus capacidades de creación, lo que implica que cada uno pueda responsabilizarse de sí mismo y realizar su proyecto personal"4. Por ende, ya no es el profesor quien va a proveer de conocimiento al estudiante, sino la persona que lo va a guiar para que encuentre, construya, organice y maneje estos conocimientos $^{5}$.

La actividad docente, entonces, ahora centrada en el estudiante, sólo logrará un aprendizaje eficaz o significativo cuando sea el mismo estudiante el que asuma la responsabilidad en la organización y desarrollo de su propio trabajo académico. En este contexto, el proyecto educativo al que adhiere la Universidad Católica del Norte (en adelante UCN):

"se hace cargo de los cambios experimentados por la sociedad chilena, la creciente inserción del país en el mundo, los avances científicos y tecnológicos, la globalización del conocimiento, el desarrollo de nuevas formas de comunicación, los modernos enfoques en materia educacional y docente, así como el impacto que todos estos factores han tenido en el sistema universitario nacional, en general, y en nuestra Casa de Estudios Superiores, en particular"6.

Este marco conceptual, que guía la formación de nuestros estudiantes, tiene como eje fundamental la educación en valores. La enseñanza centrada en el aprendizaje y la formación para la globalización, los que, a partir de la noción de responsabilidad social, articulan una serie de competencias que se pretende desarrollar y potenciar en los estudiantes, para lo cual se ha optado por un enfoque formador basado en competencias, que ha estructurado el currículo de tal manera que apunte al desarrollo integral de éstas en apoyo, además, del uso de las nuevas tecnologías de la información y comunicaciones (TIC's).

\footnotetext{
${ }^{3}$ UNESCO. Conferencia Mundial sobre Educación Superior. La educación superior en el siglo XXI: Visión y acción. París, 5 al 9 de octubre de 2008, p. 25.

${ }^{4}$ UNESCO. La educación encierra un tesoro. Informe de la UNESCO de la Comisión Internacional sobre la Educación para el siglo XXI, presidida por Jacques Delors, 1996, p. 12.

${ }^{5}$ La configuración del Espacio Europeo de Educación Superior (EEES) confirma estas premisas. Para mayor información se puede visitar el sitio: www.eees.es

${ }^{6}$ UNIVERSIDAD CATÓLICA DEL NORTE. Proyecto educativo. [Consultado 12 de julio de 2011], Disponible en web: https://online.ucn.cl/informeAutoevaluacionUCN/?rut_var=11828613\&opcion=1132\&idkey=761558298 párrafo 5.9 .
} 
Dentro de este proceso de auto-renovación ${ }^{7}$ por el que optó la UCN, la Escuela de Derecho, hoy Facultad de Ciencias Jurídicas, ha construido una malla curricular semestral de carácter flexible ${ }^{8}$, que busca incorporar la docencia del Derecho al modelo de formación por competencias recién aludido. A partir de esa perspectiva, el objetivo que persigue esta investigación es exponer nuestra experiencia en este sentido, a través de la utilización del 'método de casos', el que entendemos como uno buen ejemplo del aprendizaje significativo a través de la vivencia como una estrategia de enseñanza - aprendizaje para la disciplina del Derecho Constitucional, sus ventajas y desventajas.

\section{El aprendizaje de vivencia: la sala de clases hoy y sus nuevos desafíos}

El alumno de nuestras facultades y escuelas sufren diariamente de una dicotomía que se fragua a la luz del método tradicional de aprendizaje del derecho, el que se agudiza más aun cuando salen a la vida laboral. Esta dicotomía se basa en que nuestros alumnos saben muy bien el 'ser' del derecho: conocen conceptos, clasificaciones, naturaleza jurídica de las diversas instituciones, pero el problema está en la capacidad de aplicar dicha información. Por lo tanto, la disyuntiva está con el 'hacer', pues no tienen las destrezas desarrolladas para llevar a la práctica dicho conocimiento teórico.

Lo anterior es resultado de lo que hoy entendemos por 'aprender', que no es más que "obtener conocimiento, poseer la información correcta", esta definición es depositaria de un modelo informático del conocimiento, que responde sin lugar a dudas a un paradigma escolar $^{10}$, en donde lo esencial es preparar de la mejor manera posible el examen, prueba, control, SIMCE, Prueba de Selección Universitaria (PSU), entre otros, que se traduce en una preparación a corto plazo de las materias que se van a evaluar.

Este modelo se encuentra incompleto, pues para consumar el proceso de aprendizaje es necesario pasar de la información a la acción. Esto sin lugar a dudas tiene que ver con el perfil de estudiante que queremos tener, pues en un mercado en donde se les solicita al profesional un aprendizaje continuo para el mejor desempeño de sus capacidades, es innegable, entonces, que el abogado tiene que adquirir las capacidades que lo lleven a actuar para solucionar el conflicto jurídico que se le presente.

\footnotetext{
${ }^{7}$ IMBERNÓN, Francisco. "Un nuevo profesorado para una nueva Universidad ¿conciencia o presión?". Revista Electrónica Interuniversitaria de formación del profesorado, $\mathrm{n}^{\mathrm{o}} 38$ (edición impresa), agosto, pp 3746. [Consultado 15 de julio de 2012], Disponible en web: www.aufop.com/aufop/uploaded_files/articulos/1223490004.pdf p. 38.

${ }^{8}$ Proceso implementado a partir del 2006, gracias a la ejecución del Proyecto Mecesup UCN 0301. Ello ha derivado en un aumento progresivo de la tasa de retención de los alumnos de primer año en la escuela de Derecho, sin desatender la calidad de la enseñanza. ESCUELA DE DERECHO DE LA UNIVERSIDAD CATÓLICA DEL NORTE. Consolidación del rediseño curricular y reformulación del sistema de evaluación de competencias de las Escuelas de Derecho de la UCN, 2008, p. 11.

${ }^{9}$ KOFMAN, Fredy. Metamanagement. La nueva Conciencia de los negocios. Tomo I. Buenos Aires: Gránica, Primera Edición, 2007, p.106

${ }^{10}$ Ibid, p. 107.
} 
Según Kofman, "el círculo del aprendizaje continuo ha sido una constante entre quienes están más interesados en cambiar el mundo que en describirlo"11, y detalla este círculo al decir que tiene cuatro pasos. El primero es descubrir la realidad que se le presenta, e donde las diferencias entre lo que se experimenta y lo que quisiera que pasara efectivamente; luego hay que inventar, lo que significa que el profesional debe señalar soluciones que modifiquen lo que sucede, para adecuarlo a lo óptimo; el tercer paso es producir, lo que se traduce en poner en práctica dicha soluciones en el mundo real, y finalmente se debe reflexionar acerca de los resultados obtenidos, en donde es la base de nuevos descubrimientos lo que nuevamente nos llevará al paso número uno ${ }^{12}$.

El modelo informativo planteado solo se queda con los pasos uno y dos, no en tanto pertenece al estudiante, en este caso, ser capaz de producir una solución de manera inmediata, sino que, en el ámbito del derecho este paso se va adquiriendo con el transcurso del tiempo, y ya cuando se encuentran insertos en el mundo laboral. Lo que es peor en este sistema es que quita poder a los estudiantes, pues en vez de buscar el saber- cómo, quedan atrapados en la búsqueda del saber-qué ${ }^{13}$, lo que hoy es incompleto.

Por todo lo anterior, es que hoy se aspira a que los estudiantes de derecho, desde sus primeros años de estudio, sean capaces de ir incorporando modelos que le permitan actuar, va enfatizando el autoaprendizaje, el trabajo guiado, la conexión entre teoría y práctica, el acercamiento a la realidad laboral y el aprendizaje cooperativo ${ }^{14}$. Para lograr esto, sin lugar a dudas hay que tomar una postura frente al estilo de enseñanza. Clásicamente se diferencian tres tipos: autoritario o directivo; democrático o participativo y laissezfaire o pasivo, las que se han ido mezclando y matizando para dar paso a un docente que tiene que encontrar el justo equilibrio entre la distancia que tiene que existir con el alumno basado en el respeto, como con la proximidad de la interacción para conseguir dichas competencias ${ }^{15}$.

A fin de alcanzar este objetivo es que se propone una metodología activa como la de experimentar tanto los distintos conflictos que pueden llegar a enfrentarse en su vida laboral, así como la resolución de distintas situaciones, para hacer que esto sea incorporado en la enseñanza- aprendizaje del Derecho Constitucional, lo que desde la filosofía se ha denominado la vivencia.

\footnotetext{
${ }^{11}$ Ibíd.

${ }^{12}$ Ibid, pp. 107 y 108.

${ }^{13}$ Ibid, p. 110.

${ }^{14}$ CARPIO, Carmen, "Métodos de Enseñanza- Aprendizaje Aplicables en Magisterio en el Marco del Espacio Europeo de Educación Superior”. Revista Docencia e Investigación Universidad de Castilla-La Mancha. 2008, № p.2 [Consultado 7 de noviembre de 2012]. Disponible en: www.uclm.es/varios/revistas/docenciaeinvestigacion/numero8.asp

${ }^{15}$ Ibíd.
} 


\section{La metodología de vivencias}

La vivencia deriva del latín viventia que significa 'experiencia de vida'. Uno de los primeros autores que investigó el sentido de este concepto fue el filósofo historicista Wilhelm Dilthey, quien al estudiar el término en alemán erlebnis (experiencia, vivencia) señaló que era "algo revelado en el complejo psíquico dado en la experiencia interna de un modo de existir la realidad para un individuo" ${ }^{16}$. En otras palabras, este concepto se hace patente al estar presente en el aquí y ahora para poder, desde esa perspectiva, adquirir los elementos necesarios para experimentar lo necesario en el desarrollo de la vida, a través de vivencias sustanciales y significativas.

El vivir para este autor, en sí mismo, es el mayor aprendizaje que un ser humano puede tener, y esto significa nada más que hay que dejar experimentar, dejar obrar y actuar con el apoyo de la aprehensión inmediata, la intuición y la fuerza del universo. Por tanto, cada acto del ser vivo se encuentra inmerso en la particularidad por un lado, pero por la universalidad de dicha acción con la conexión del todo por otro ${ }^{17}$.

Sin lugar a dudas cómo se vivencia una determinada situación dependerá ostensiblemente del tipo de ciencias a la cual nos enfrentemos, pues podemos estar ante corrientes sintéticas o analíticas. Así:

En cuanto a las primeras, se consideran aquéllas que producen conceptualmente su objeto y cuya validez depende de la organización interna de la misma filosofía o campos de saber respectivos como es el caso de la matemática. En cambio, las filosofías analíticas implican la experiencia como fuente y base del conocimiento, buscan en la descripción o análisis de los 'hechos' la resolución del objeto dentro de los elementos o condiciones que permiten entenderlo; pues en la experiencia, o intuición sensible, los objetos son datos que sólo pueden ser analizados y, por consiguiente, su elección y análisis resolutivo constituyen el problema que atañe a la determinación del campo donde se pueden o deben obtener esos mismos datos ${ }^{18}$.

Y en este último campo se inserta el mundo del Derecho, o de las Ciencias Jurídicas.

Para Dilthey lo propiamente humano radicaba de forma precisa en la actividad racional que se manifestaba y desenvolvía en la vivencia, la expresión y la comprensión, en tanto estas últimas son tanto basamento estructural de las ciencias del espíritu como categorías metodológicas para investigar lo humano, lo que comienza del entendido que el conocimiento se construye desde la relación del sujeto y objeto ${ }^{19}$. Esto lo llevó a reconocer en la intuición un medio de conocimiento, tanto por su función instrumental como por la

\footnotetext{
16 TORO, Rolando. Biodanza. Santiago de Chile: Cuarto Propio. 2009, pp. 32- 33.

${ }^{17}$ AMARAL, María Nazaré de Camargo Pacheco. "Dilthey y la educación". Revista Educación y Pedagogía, 2000, Número 26-27, Vol. XII, Antioquia, p. 118.

${ }^{18}$ PAULIN, Georgina; HORTA, Julio; SIADE, Gabriel, "La vivencia y su análisis: consideraciones breves sobre las nociones objeto-sujeto en el universo discursivo del mundo cultural". Revista Mexicana de Ciencias Políticas y Sociales. 2009, N 205, Universidad Nacional Autónoma de México, México, D.F., p.20

${ }^{19}$ Ibíd. p. 25.
} 
esencia práctica del ser humano cuyas funciones dominantes eran el sentimiento, la voluntad y la representación ${ }^{20}$, lo que va poniendo al lado del conocimiento discursivo racional, el intuitivo -racional, por su función instrumental y por la esencia práctica del ser humano.

La vivencia, entonces, es un curso de experiencia y tiene un componente temporal, en el sentido que lo que se experimenta es en el aquí y el ahora, pero en medida que va teniendo en consideración lo ya vivido, lo que el autor llama vivencia del recuerdo, y lo que se va vivir, denominado vivencia del futuro ${ }^{21}$.

El proceso de aprendizaje tiene como punto de partida, la conexión o unidad entre el recuerdo y la vivencia presente, y lo que une estos dos conceptos es la conciencia, que:

"se concibe como instrumento de conocimiento y también como facultad representativa, mediante la cual se establece la relación entre el hombre y algo externo a él. De esta manera, en el conocimiento, como fenómeno de conciencia, se produce una relación de trascendencia entre sujeto y el objeto de donde, en el primero, surge una imagen, una idea por medio de la cual la conciencia (intuitiva, discursiva, intencional) aprehende, representa, piensa el objeto que, no obstante permanecer trascendente al sujeto, determina la imagen o el contenido del pensamiento. En cambio, el sujeto, a través de su conciencia cognoscente, tiene la posibilidad de modificar no sólo esos contenidos sino, en su proyección práctica, de orientar transformaciones en lo dado y producir nuevos objetos" ${ }^{22}$.

Esto ocurre puesto que en la vivencia de determinadas situaciones dentro de la sala de clases, el alumno utiliza todo el bagaje de los conocimientos preexistentes y los aplica a la resolución concreta del conflicto, no solo a través de la razón, sino que también va utilizando la intuición y lo que muchas veces llamamos como el 'olfato jurídico'. Así, cuando nuevamente se ven enfrentados a una situación con características similares ellos traen de regreso al presente lo que ya experimentaron en la clase para dar nuevas y renovadas soluciones.

Lo anterior se explica en los diversos pasos que utiliza la persona para llevar a cabo este proceso, y en este caso, a los alumnos de Derecho Constitucional, a construir su vivencia. En primer lugar el paso mental necesario es la captación de lo dado, en el acto de concebir la situación planteada, solo en tanto la compara y va estableciendo las igualdades y diferencias contenidas en la percepción de las circunstancias. Luego viene una operación abstractiva de la inteligencia, en virtud de la cual, lo observado es elevado a una idea o concepto objetivo, para en último paso dar pie a la actividad judicativa o de síntesis, en virtud de la cual las operaciones de comparar, separar y unir permiten que lo captado se haga explícito en el pensamiento ${ }^{23}$.

\footnotetext{
${ }^{20}$ Ibíd.

${ }^{21}$ Ibíd.

${ }^{22}$ Ibíd. p. 26.

${ }^{23}$ Ibíd.
} 
Luego aparece un nuevo tipo de relación mental: la representación recordada, en tanto lo que se hizo explícito en el paso anterior ahora es reproducido ${ }^{24}$ en la nueva situación. Esta etapa es previa al pensamiento discursivo, lo que se vincula el cómo se aplica lo aprendido a nuevos contextos, lo que va generando diversas soluciones, o en términos de Kofman, el producir.

Lo importante de todo este tránsito en la vivencia es que por el proceso de abstracción que ocurre en ella, lo que es cierto para el caso concreto que es seleccionado por el pensamiento y puede ser utilizado en una serie de situaciones más o menos homogéneas de modo tal que lo vivido se va volviendo una especie de regla general.

Este método se aplica a las ciencias de la naturaleza como a las ciencias del espíritu, en donde es lo vivencial aquello de carácter esencial en esta última, pues:

"no se buscan razones explicativas hipotéticas pues las causas de esta mismidad se experimentan en lo vivo, sin embargo, la captación intelectual de la vivencia requiere hacer explícito lo que en su concreción y variabilidad se haya implícito, esto es, se necesita pasar de la subjetividad de la vivencia a la objetivación de la vida" 25 .

\section{Aplicación de la vivencia en el Derecho Constitucional}

La enseñanza del Derecho Constitucional posee ciertas particularidades que lo distinguen, como por ejemplo que se imparte desde el ingreso a la carrera a estudiantes que entran por primera vez a la universidad, y que en su mayoría provienen de un sistema educacional deficiente ${ }^{26}$. Así, es la disciplina del derecho a través de la cual se regula normativamente el ejercicio del poder político y se protege el ejercicio de los derechos fundamentales, ambos son elementos que poseen una importante carga ideológica ${ }^{27}$. Se trata, además, de una materia con un alto contenido axiológico y ético, a nuestro juicio trascendental en la formación del profesional abogado; y, la estructura de principio que poseen las normas constitucionales, conlleva una dificultad mayor para su comprensión e interpretación por parte del estudiante de Derecho que se encuentra en los primeros niveles de la formación profesional, lo cual exige habilidades de comprensión lectora y de expresión oral y escrita que no son en rigor una característica promedio en el estudiantado que ingresa a la universidad. En definitiva, el Derecho Constitucional "aunque sea una más de las disciplinas jurídicas, no es una disciplina como las demás (...) Por eso, no sólo cabe afirmar que nuestro ámbito de estudio se sitúa en lo jurídico, sino que también determina la

${ }^{24}$ Ibíd. p, 27.

${ }^{25}$ Ibíd.

${ }^{26}$ Un $43 \%$ de los colegios secundarios en Chile no imparte el currículo completo. Fuente: Informe sobre educación de la OCDE. [Consultado 21 de Marzo de 2012]. Disponible en web: http://mt.educarchile.cl/MT/jjbrunner/archives/La\%20Educaci\%F3n\%20Superior\%20en\%20Chile0407.pdf.

${ }^{27}$ BASSA, Jaime. "Neutralidad política y enseñanza del Derecho Constitucional en Chile". En Henríquez, Miriam (coord.): Perspectiva del Derecho Constitucional desde el mirador del Bicentenario, Santiago: Librotecnia, 2010, pp. $271-288$, p. 271. 
propia definición jurídica del resto. Y, no obstante, no debemos desdeñar la realidad y lo político"28.

Es por ello que resulta esencial que los alumnos puedan experimentar las diversas situaciones que construyen dicha realidad, a través una serie de ejercicios prácticos que los hagan sentir y vivir lo que significa la construcción del sistema en el cual nos encontramos inmersos.

A partir de este punto, es necesario es diferenciar dos tipos de vivencias: aquellas que tienen como objetivo que el estudiante viva, en primera persona, algunas de las problemáticas o situaciones que a futuro le va corresponder entregar su consejo profesional a fin de crear cierta sensibilidad frente al problema dado. Y, por otro lado, aquellas que posicionan al estudiante como futuro abogado donde la experiencia viene dada por la resolución del caso concreto, que desde esta perspectiva se relaciona directamente con el método de casos y el aprendizaje basado en problemas.

La primera de estas es una opción novedosa y que presenta mayores retos al momento de aplicarla en las salas de clases, pues se trata de colocar al estudiante frente a la situación de hacerse responsable en el acontecer cotidiano, y de la misma manera, que sea capaz de darse cuenta del peso de la realidad y su rol como abogado en la misma. Es por esto que durante los cursos se realizan diversas actividades como la que se detallan a continuación.

\section{El método activo para vivenciar}

Las cuatro materias que contempla el Derecho Constitucional (teoría constitucional, teoría de los derechos fundamentales, sistema constitucional chileno: parte orgánica y parte dogmática) son articuladas con un orden lógico de menos a más, esto significa que cada una de las competencias que se pretende desarrollar son profundizadas en cada nivel, con el fin de lograr que el alumno adquiera el denominado "criterio jurídico' ${ }^{29}$ que como carrera hemos declarado que el alumno debiera adquirir y desarrollar en el transcurso de cinco años.

\footnotetext{
${ }^{28}$ SANZ MORENO, José Antonio. "Derecho Constitucional, objeto y método: ¿Siglo XX o siglo XXI". comunicación enviada a la Jornada sobre orientación y método del Derecho Constitucional, Inédito. 2008 . p 2. [Consulta 30 de junio de 2011] Disponible en web: http://www.uned.es/dpto-derechopolitico/Comunicacion_JA_Sanz.pdf

${ }^{29}$ Que implica el dominio de dos competencias fundamentales: la primera se identifica como el "posicionamiento frente al asunto de relevancia jurídica, que conlleva como elementos: 1) Comprender, analizar e interpretar el asunto de relevancia jurídica; y 2) determinar posibilidades de solución para el asunto de relevancia jurídica". La segunda competencia se identifica como "ejecución de instrumentos que dan solución al asunto de relevancia jurídica", el que conlleva como elementos: 1) identificar y decidir actos para materializar la solución del asunto de relevancia jurídica; y 2) diseñar y ejecutar estrategias e instrumentos para dar solución al asunto de relevancia jurídica. ESCUELAS DE DERECHO DE LA UNIVERSIDAD CATÓLICA DEL NORTE. Informe de autoevaluación. Inédito, año 2012, pp. 8 - 14.
} 
Es por ello que en la primera clase de Derecho Constitucional I firmamos una suerte de compromiso, el cual más tarde ellos conocen como contrato social, con el fin de explicitar los objetivos que tiene no solo la asignatura sino también la metodología que empleamos, lo que implica darles a conocer de forma clara los fines que persigue la materia de Derecho Constitucional; los derechos que ellos tendrán como estudiantes de esta asignatura; las reglas que regulan la convivencia del grupo; los deberes que asumimos; la programación semestral de las actividades y de todas las evaluaciones (incluimos en ello también los criterios o pautas de evaluación, tanto de actividades escritas como orales). Esta simple actuación los acerca a nociones fundamentales de democracia y ejercicio de derechos, pues la postura del docente debe estar abierta a aceptar críticas o cuestionamientos que son válidos desde la perspectiva del estudiante. El logro de un consenso es el punto de partida para la generación de un acuerdo marco que incentiva la responsabilidad y respeto por los logros alcanzados.

Asimismo, durante el primer curso se ven materias como teoría del Estado, Gobierno, Constitución y Democracia, lo importante es que el alumno sea capaz de percibir cómo se interconectan los distintos elementos para dar paso a la realidad política que hoy tenemos, y por eso se parte desde el núcleo más elemental de la misma haciendo que los estudiantes sean capaces de construir una sociedad política determinada, a partir de ciertos supuestos de hecho dados, que configura, de esa manera, un Estado con su propia realidad histórica, una Constitución, un Gobierno con sus diversos órganos y competencias, para finalmente construir su sistema democrático, que va articulando los diversos elementos de manera coherente y con sentido.

Este método activo para vivenciar, como le hemos denominado, en tanto estrategia de enseñanza - aprendizaje, se sustenta en el trabajo grupal, de 3 ó 4 personas. Cada grupo va conformando semana tras semana un portafolio de actividades que les permite cada cierto tiempo autoevaluar su propio desempeño y evaluar el de sus compañeros (co-evaluación), así como también los lleva al logro de los objetivos académicos planteados de manera gradual.

La idea es que cada grupo asuma un rol activo en la configuración de un sistema político nuevo, lo que les demanda la aplicación de conocimientos de teoría política y constitucional en cada una de las decisiones que ellos adoptan. Cada opción debe ser necesariamente fundamentada y además coherente con decisiones adoptadas en etapas previas, lo que impone además la necesidad de estar permanentemente informados sobre cuestiones de contingencia política, social y económica.

Por lo tanto, cada semana se van incorporando elementos o actores nuevos al sistema político que ellos están creando, a través de ejercicios: preguntas y respuestas (que principalmente sirven para fijar contenidos dogmáticos); elaboración de informes (que buscan aplicar la teoría a la realidad que ellos están elaborando); exposiciones; actividades más lúdicas como bachilleratos, "carreras del saber" e instrucción entre pares, lo que les permite desplegar una serie de competencias que necesariamente debemos evaluar semanalmente, sea en aula o a través de trabajos para la casa. 
La programación de todas actividades es previa, se entrega al inicio del semestre, y a su vez cada actividad es acumulativa e incorpora lo que el alumno ya sabe. A pesar de ello, también nos permitimos una cuota de flexibilidad ya que cada curso es distinto de otro, lo que a veces demanda algunos cambios que siempre y en todo caso llevamos a la "consulta popular" puesto que se trata de modificaciones al contrato social que funda la asignatura.

\section{La vivencia y el método de casos}

El método de casos es originario de Estados Unidos y es el que inspira la docencia de las Ciencias Jurídicas en ese país ${ }^{30}$. Aquel consiste en la descripción de una situación concreta con finalidades pedagógicas para aprender o perfeccionarse en algún campo determinado. El caso se propone a un grupo-clase para que individual y colectivamente lo sometan al análisis y a la toma de decisiones. Al utilizar el método del caso se pretende que los alumnos estudien la situación, definan los problemas, lleguen a sus propias conclusiones sobre las acciones que habría que emprender, contrasten ideas, las defiendan y las reelaboren con nuevas aportaciones y que generalmente plantea problemas divergentes, pues éste no tiene una única solución.

De igual manera, este sistema contribuye al desarrollo de habilidades en la medida en que vincula al estudiante con hechos reales que le permiten desarrollar su propio análisis y adoptar una solución que considere adecuada. En este sentido, los casos presentan las siguientes características:

a) Pueden replicar situaciones de crítica, de riesgo o incertidumbre, que son propios de la vida real;

b) Contribuyen a dar un enfoque práctico y pragmático a situaciones diversas y variadas;

c) Entregan al estudiante la responsabilidad de su propio aprendizaje, y lo motivan a permanecer informado y activo en su profesión;

d) Requiere de una participación activa y favorecen al desarrollo de habilidades de comunicación.

Cuando el alumno llega al último nivel, Derecho Constitucional IV, la asignatura se imparte sólo en base al análisis de jurisprudencia constitucional relacionada con los distintos derechos consagrados en la Carta, así como jurisprudencia internacional que nos permite efectuar un trabajo de comparación de los criterios interpretativos utilizados en ambas sedes jurisdiccionales.

Con este planteamiento es posible no solo que el alumno desarrolle sus capacidades cognitivas que indudablemente son importantes, pero además constatamos el progresivo

\footnotetext{
${ }^{30}$ SERNA DE LA GARZA, José María. “Apuntes sobre las opciones de cambio en la metodología de la enseñanza del Derecho en México". Boletín Mexicano de Derecho Comparado, nueva serie, año XXXVII, N 111, septiembre - diciembre de 2004, Mexico D.F., pp. 1047 - 1082, p. 1048.
} 
despliegue de habilidades que sustentan su conocimiento y por sobre todo que lo comprometen con un ideal de Estado constitucional y democrático de Derecho.

La implementación de esta estrategia nos ha permitido constatar que el aprendizaje de los alumnos se construye a partir de un 'pensamiento divergente', en donde las soluciones novedosas (sobre todo para asuntos o temas nuevos para el Derecho, como Internet, el progreso científico, la globalización, etc.) siempre deben ser bienvenidas, vale decir, ya no debiera importar tanto el resultado al que arriba el alumno ante una determinada situación, sino más bien el proceso a través del cual llegó a ese resultado. Desde esta experiencia, nos parece que en este ambiente es donde florece de la mejor manera el mentado criterio jurídico del que hacemos gala los abogados.

Junto a ello, otra manera de 'vivir' los distintos conflictos que se evidencian a la luz de los derechos fundamentales son los 'juegos de roles'. En este caso a los alumnos se les pide que actúen diversas situaciones de la vida real con el objeto de que perciban los distintos escenarios que pueden llegar a experimentar sus futuros representados, de modo tal que ellos sean capaces de ponerse en el lugar del otro y puedan experimentar empatía por el conflicto que está afectando a una persona determinada.

El escenario es entregado por un caso que determina los hechos de una situación real, pues ha sido fallada por un tribunal. Al recibirlo, los estudiantes en grupo crean un libreto que les permite actuar una pequeña obra teatral que relata los acontecimientos que originaron un conflicto de derechos fundamentales.

Paralelamente, otro grupo prepara un libreto a partir de los mismos hechos, pero que contiene una vivencia esta vez respetuosa del ejercicio de los derechos que, por ende, llega a un resultado jurídico diferente.

Luego de actuar ambas situaciones, se origina un debate en donde se contrastan las diferentes posturas, lo que permite que dialécticamente se pueda argumentar a favor o en contra de distintas interpretaciones de las normas constitucionales, y así van contrastando los resultados jurisprudenciales con la opinión fundamentada de los alumnos.

\section{Resultados del modelo de aprendizaje basado en vivencias}

La construcción de este sistema de enseñanza-aprendizaje no se encontraría completo si no contáramos con la opinión de los estudiantes que vivencian esta novedosa forma de aprender-cómo.

Es por ello que al concluir los cuatro cursos de Derecho Constitucional, se les aplica una encuesta que cuenta con 19 afirmaciones que abarcan los siguientes criterios: 
a) Aquellos que se relacionan con la aplicación de esta metodología a otras áreas del Derecho.

b) Aquellos que dicen relación con la aplicación de esta metodología y su relevancia para la formación profesional.

c) Aquellos que tienen que ver con la adquisición de competencias transversales, como por ejemplo expresión oral y escrita, uso de bases de datos, investigación, etc.

d) Aquellas que buscan evidenciar el grado de satisfacción del alumno con el uso de esta metodología.

En efecto, podemos señalar que el 100\% de los alumnos que están cursando el último nivel de la asignatura está contento con las diversas metodologías empleadas a lo largo de los cursos de Derecho Constitucional, así como con el trabajo cooperativo, con la mejora en sus habilidades orales y escritas, por lo que se sienten más preparados para entrar al mundo laboral y le gustaría repetir este tipo de experiencias en otras disciplinas jurídicas.

Por otra parte, también constatamos que un $80 \%$ de los alumnos estima que invierte mucho tiempo fuera de clases para la confección de los trabajos en grupos, lo que sin duda es un elemento a considerar para el futuro.

Junto a ello, un 33\% declara que hubiese preferido trabajar solo, y un 35\% señala haber tenido conflicto al interior de su grupo, lo que por una parte demanda una mayor atención del docente y de su equipo de ayudantes; pero por otro, nos permite inferir la existencia de un grado de incapacidad para resolver conflictos con sus pares y para adaptarse a nuevas situaciones que les demandan algunas habilidades sociales que no están acostumbrados a desplegar.

\section{Ventajas y desventajas de la metodología empleada}

Conforme con lo anterior, creemos que es necesario adaptar la clase tradicional a estas nuevas ideas, pues los sistemas educativos debieran ser orgánicos y no industrializados tal como lo ha señala Sir Ken Robinson ${ }^{31}$, es decir, debemos procurar crear en el aula el ambiente adecuado para que cada estudiante primero logre averiguar cuáles son sus talentos y luego pueda desarrollarlos a lo largo de su existencia.

Ahora bien, como señalamos anteriormente, la metodología descrita cuenta con un alto grado de aceptación por parte de los alumnos, aunque debemos reconocer también que no todos los estudiantes están capacitados para acceder al aprendizaje de esta forma, ya que están muy acostumbrados a permanecer pasivos dentro de la sala de clases, lo que también puede ser un obstáculo y en algunas ocasiones nos demanda un alto grado de histrionismo a

\footnotetext{
${ }^{31}$ ROBINSON, Ken. The element: how finding your passion changes everything. London: Penguin Books Limited, 2009, 288 pp.
} 
fin de incentivarlos lo máximo posible, lo que implica una gran dedicación de parte del equipo de personas que trabaja en esta tarea.

Sin lugar a dudas, todo es perfectible y cada fin de semestre el equipo se reúne y vamos discutiendo los pros y los contras que hemos encontrado, con el fin de retroalimentarnos e implementar cambios en nuestro modelo. Es así que como críticas a este sistema podemos decir que éstas se fundamentan en cuestiones logísticas, como por ejemplo:

a) El número de alumnos en cada curso. Esta metodología funciona mejor en cursos pequeños, pero en esa decisión, que es institucional, está en juego un asunto de corte financiero y presupuestario que es importante para la Universidad, de hecho entra una gran cantidad de alumnos a primer año y aun cuando se trate de un curso que es impartido en formato paralelo por dos profesores, el número de inscritos es muy grande ${ }^{32}$.

b) Carencia de competencias al ingreso, cuestión que tiene que ver con el sistema educativo chileno en general y que ahora mismo se discute a nivel nacional.

c) Cuestiones de infraestructura, no contamos con salas de clases que incentiven el trabajo en grupo, de hecho la mayoría de ellas están construidas para la clase conferencia.

d) La alta demanda de tiempo que tiene la implementación de este modelo, que necesita de un grupo de personas cohesionado y muy comunicado entre sus integrantes.

e) No todas las asignaturas de la malla cuentan con estrategias de enseñanza aprendizaje activa, entonces lo que puede lograrse con mucho esfuerzo puede perderse fácilmente si no existe una continuidad en este sentido en las demás asignaturas de la carrera. Allí cabe destacar por ejemplo que nuestra Facultad no cuenta con una unidad de apoyo pedagógico como existen en otras Facultades, que permita implementar este tipo de iniciativas a lo largo de toda la malla.

f) Las necesidad de formar los grupos de ayudantes que existen en las Escuelas también me parece vital, sobre todo si pensamos en la necesidad del recambio y para ello es necesario invertir en capacitaciones o incentivos para la investigación y para que estos alumnos deseen profundizar en su formación con estudios de posgrado.

Por último, sobre los aspectos positivos del modelo que utilizamos, me parece que lo hemos dicho, puesto que su utilización permite "una enseñanza del Derecho Constitucional entendido como algo vivo, alejada de dogmatismos y dirigida a la formación de hombres y mujeres flexibles, críticos, suficientemente militantes con los valores constitucionales pero en ningún caso prisioneros de una "geometría" que es imposible en una rama del Derecho tan impregnada de política... y de vida"33.

\footnotetext{
${ }^{32}$ A modo de ejemplo, en el 2011 el curso de Derecho Constitucional I contó con 53 alumnos y el año 2012 con 46.

${ }^{33}$ SALAZAR BENÍTEZ, Octavio. "Estrategias y herramientas para la enseñanza del Derecho constitucional". En Docencia y Derecho, año 2010, N $\mathrm{N}^{\mathrm{N}}$ 1, p. 8 [Consultado 20 de marzo de 2012], Disponible en web: http://ww.uco.es/docencia_derecho/index.php/reduca/article/view/30
} 
Como lo hemos señalado, la utilización de estas estrategias nos permiten evaluar el proceso de aprendizaje de los estudiantes, desde las tres perspectivas fundamentales en las que se enfoca el saber: conocimiento, habilidades y actitudes o valores que son fundamentales para el ejercicio de nuestra profesión en una sociedad del siglo XXI.

\section{Referencias bibliográficas}

AMARAL, María Nazaré de Camargo Pacheco. "Dilthey y la educación". Revista de Educación y Pedagogía. 2000, N²6-27, Vol XII, Antioquia, pp. 107-121.

BASSA, Jaime. "Neutralidad política y enseñanza del Derecho Constitucional en Chile". En Henríquez, Miriam (coord.). Perspectiva del Derecho Constitucional desde el mirador del Bicentenario. Santiago: Librotecnia, 2010, pp. $271-288$.

CARPIO, Carmen. "Métodos de Enseñanza- Aprendizaje Aplicables en Magisterio en el Marco del Espacio Europeo de Educación Superior". Revista Docencia e Investigación Universidad de Castilla-La Mancha. 2008, No 18, [Consultado 7 de noviembre de 2012] Disponible en web: www.uclm.es/varios/revistas/docenciaeinvestigacion/numero8.asp

ESCUELAS DE DERECHO DE LA UNIVERSIDAD CATÓLICA DEL NORTE. Consolidación del rediseño curricular y reformulación del sistema de evaluación de competencias de las Escuelas de Derecho de la UCN, Proyecto Mecesup II, 2008, 254pp.

IMBERNÓN, F. "Un nuevo profesorado para una nueva Universidad ¿conciencia o presión?”. Revista Electrónica Interuniversitaria de formación del profesorado. 2000, n 38 pp 37-46. [Consultado 15 de julio de 2011] Disponible en web: www.aufop.com/aufop/uploaded_files/articulos/1223490004.pdf

KOFMAN, Fredy. "Metamanagement. La nueva Conciencia de los negocios", Tomo I, Granica. Buenos Aires: Granica, Primera Edición, 2007, 358 pp.

PAULIN, Georgina; HORTA, Julio; SIADE, Gabriel, "La vivencia y su análisis: consideraciones breves sobre las nociones objeto-sujeto en el universo discursivo del mundo cultural". Revista Mexicana de Ciencias Políticas y Sociales. 2009, N ${ }^{\circ} 205$, Universidad Nacional Autónoma de México, México, D.F., pp. 15-35.

ROBINSON, Ken. The element: how finding your passion changes everything, London: Penguin Books Limited, 2009, 288 pp.

SALAZAR BENÍTEZ, Octavio. "Estrategias y herramientas para la enseñanza del Derecho constitucional". En Docencia y Derecho, año 2010, $\mathrm{N}^{\mathrm{o}} 1$. Disponible en: http://ww.uco.es/docencia_derecho/index.php/reduca/article/view/30 [Fecha de visita: 20 de marzo de 2012], p. 8. 
SANZ MORENO, José Antonio. Derecho Constitucional, objeto y método: ¿Siglo XX o siglo XXI. Comunicación enviada a la Jornada sobre orientación y método del Derecho Constitucional. Inédito. [Consultado 30 de Julio de 2011] Disponible en web: http://www.ned.es/dpto-derecho-politico/comunicacion_ja_sanz.pdf

SERNA DE LA GARZA, José María (2004). "Apuntes sobre las opciones de cambio en la metodología de la enseñanza del Derecho en México". Boletín Mexicano de Derecho Comparado. nueva serie, año XXXVII, No 111, septiembre - diciembre de 2004, México D.F., pp. 1047 - 1082.

TORO, Rolando. Biodanza. $3^{\text {a }}$ edición. Santiago: Cuarto Propio, 2009, 294 pp.

Unesco. Conferencia Mundial sobre Educación Superior. La educación superior en el siglo XXI: Visión y acción. Celebrada el 9 de octubre, año 2008. [Consultado 12 de Julio de 2011]. Disponible en web: http://www.unesco.org/education/educprog/wche/declaration_spa.htm

Unesco. La educación encierra un tesoro. Informe de la UNESCO de la Comisión Internacional sobre la Educación para el siglo XXI, presidida por Jacques Delors. Madrid: Santillana, 1996, 44 pp.

Universidad Católica del Norte. Proyecto educativo. [Consultado 12 de julio de 2011]. Disponible en web: https://online.ucn.cl/informeAutoevaluacionUCN/?rut_var=11828613\&opcion=1132\&idke $\mathrm{y}=761558298$ 